Delft University of Technology

\title{
Dual-Gate Fet-Based Charge Sensor Enhanced by In-Situ Electrode Decoration in a MEMS Organs-On-Chip Platform
}

Aydogmus, Hande; van Ginkel, H. Joost; Galiti, Anna-Danai ; Hu, Michel; Frimat, Jean-Philippe; van den Maagdenberg, Arn ; Zhang, GuoQi; Mastrangeli, Massimo ; Sarro, Pasqualina M.

DOI

10.1109/Transducers50396.2021.9495393

\section{Publication date}

2021

Document Version

Final published version

Published in

21st International Conference on Solid-State Sensors, Actuators and Microsystems, TRANSDUCERS 2021

\section{Citation (APA)}

Aydogmus, H., van Ginkel, H. J., Galiti, A-D., Hu, M., Frimat, J-P., van den Maagdenberg, A., Zhang, G., Mastrangeli, M., \& Sarro, P. M. (2021). Dual-Gate Fet-Based Charge Sensor Enhanced by In-Situ Electrode Decoration in a MEMS Organs-On-Chip Platform. In 21st International Conference on Solid-State Sensors, Actuators and Microsystems, TRANSDUCERS 2021 (pp. 180-183). [9495393] (21st International Conference on Solid-State Sensors, Actuators and Microsystems, TRANSDUCERS 2021). IEEE . https://doi.org/10.1109/Transducers50396.2021.9495393

Important note

To cite this publication, please use the final published version (if applicable).

Please check the document version above.

\section{Copyright}

Other than for strictly personal use, it is not permitted to download, forward or distribute the text or part of it, without the consent of the author(s) and/or copyright holder(s), unless the work is under an open content license such as Creative Commons.

Takedown policy

Please contact us and provide details if you believe this document breaches copyrights.

We will remove access to the work immediately and investigate your claim. 
Green Open Access added to TU Delft Institutional Repository

'You share, we take care!' - Taverne project

https://www.openaccess.nl/en/you-share-we-take-care

Otherwise as indicated in the copyright section: the publisher is the copyright holder of this work and the author uses the Dutch legislation to make this work public. 


\section{DUAL-GATE FET-BASED CHARGE SENSOR ENHANCED BY IN-SITU ELECTRODE DECORATION IN A MEMS ORGANS-ON-CHIP PLATFORM}

Hande Aydogmus $^{1}$, H. Joost van Ginkel ${ }^{1}$, Anna-Danai Galiti ${ }^{1}$, Michel Hu ${ }^{2,3}$, Jean-Philippe Frimat ${ }^{2,3}$, Arn van den Maagdenberg, ${ }^{2,3}$, GuoQi Zhang ${ }^{1}$, Massimo Mastrangeli ${ }^{1}$, and Pasqualina M. Sarro ${ }^{1}$

${ }^{1}$ ECTM, Department of Microelectronics, Delft University of Technology, the Netherlands.

${ }^{2}$ Department of Human Genetics, Leiden University Medical Centre, the Netherlands.

${ }^{3}$ Department of Neurology, Leiden University Medical Centre, the Netherlands.

\begin{abstract}
Continuous monitoring of tissue microphysiology is a key enabling feature of the organ-on-chip (OoC) approach for drug screening and disease modeling. Sensing charged species in $\mathrm{OoC}$ tissue microenvironments is thereby essential. However, the inherently small (i.e., $\mathrm{cm}$ ) size of OoC devices poses the challenging requirement to integrate miniaturized and highly sensitive in situ charge sensing components to maximize signal extraction from small volumes (nL to $\mu \mathrm{L}$ range) of media used in these devices. Here we meet this need by presenting a novel dual-gate field-effect transistor-based charge sensor integrated within an optically transparent microelectromechanical (MEM) OoC device. Post-process mask-less decoration of Ti sensing electrodes by spark-ablated Au nanoparticle films significantly increases the effective electrode surface area and thus sensor sensitivity while retaining the CMOS-compatibility of the wafer-level fabrication process. We validate the biocompatibility of the sensor and its selective response to poly-D-lysine and $\mathrm{KCl}$, and provide a perspective on monitoring cultures and differentiation of hiPSC-derived cortical neurons on our OoC device.
\end{abstract}

\section{KEYWORDS}

Biosensor, charge sensing, electrodes, microfabrication, organ-on-chip, spark ablation, neurons

\section{INTRODUCTION}

Organs-on-chip (OoCs) are dynamic tissue culture devices which mimic microphysiological environments to recapitulate in vivo-like organ functions in vitro and thus enhance the efficiency of drug development and disease modelling [1]. Among the key features distinguishing OoCs from established tissue culture technologies, automated monitoring of biological cues, such as charged species, is essential to reduce user intervention and improve system's ease of use and reliability [2]. In this respect, dynamic electric sensing can meet user needs without recurring to terminal optical labeling and microscopy techniques, which deteriorate cells' wellbeing.

To provide compact charge sensing solutions in OoCs, the integration of a floating gate [3-5] field-effect transistor (FG-FET)-based electrochemical sensor within a microelectromechanical OoC device was previously demonstrated [6]. Prior examples of FET-based bio-chemical sensing include tracking metabolic activity of neurons [7-8] and of $\mathrm{pH}$ [9]. Our hybrid OoC combines in a single device the benefit of soft polymeric substrates, suitable for tissue culturing under physiologically relevant conditions, with that of extremely compact and inherently signal-amplifying electronic sensing units, uniquely enabled by silicon-based microfabrication [10]. Building on that, in this paper, we

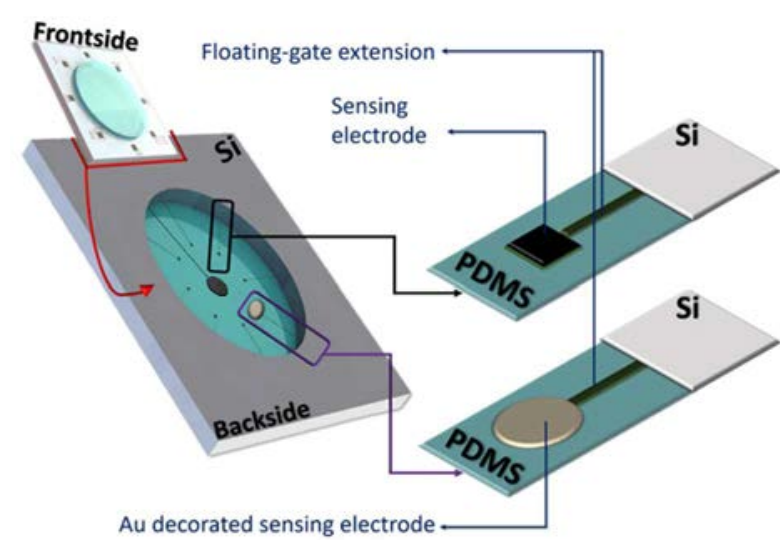

Figure 1: Schematic of the MEM OoC device with integrated FET-based charge sensor. Right insets show sensing electrodes over the suspended membrane without (top) and with (bottom) decoration with Au film.

show significant enhancement in sensitivity and in selective response of the FG-FET-based charge sensor. Both breakthroughs were enabled by the introduction of a novel dual-control-gate configuration and by in situ decoration of the sensing electrode surface with nanoporous $\mathrm{Au}$ thin films (Fig. 1).

The OoC device is fabricated by means of a mostly CMOS-compatible co-fabrication process. The charge sensor therein replaces the function of an external reference electrode with that of a pair of control gates $\left(\mathrm{CG}_{1}\right.$ and $\left.\mathrm{CG}_{2}\right)$ capacitively coupled to the floating gate. An extension of the FG reaches over the suspended polymer membrane, representing the optically accessible tissue culturing area, and terminates with a sensing electrode. The charge stored in the dielectric layer (silicon oxide) between $\mathrm{CG}_{1}$ and $\mathrm{FG}$ sets the working point of the transistor, whereas the electrolyte bridging $\mathrm{CG}_{2}$ and $\mathrm{FG}$ serves as dielectric as well as solution-under-test (SOT). Biasing $\mathrm{CG}_{2}$ provides electric field towards the sensing electrode at the tip of the FG extension. This promotes the binding of charged species on the sensing surface and thus provides higher sensitivity (Fig. 2(a)). In turn, the charges in close proximity of the sensing electrode can be continuously detected by monitoring the drain current $\left(\mathrm{I}_{\mathrm{D}}\right)$ of the transistor.

Charge sensing exploits the electrical double layer [11], as it involves (reversible) binding by charged species in solution to sites available at the sensing surface. Therefore, to further enhance sensitivity we aimed at expanding the effective electrode surface and increasing the number of available binding sites by means of surface structuring with Au. Due to its inherent biocompatibility and inertness, $\mathrm{Au}$ has been widely used for biosensing applications as a 

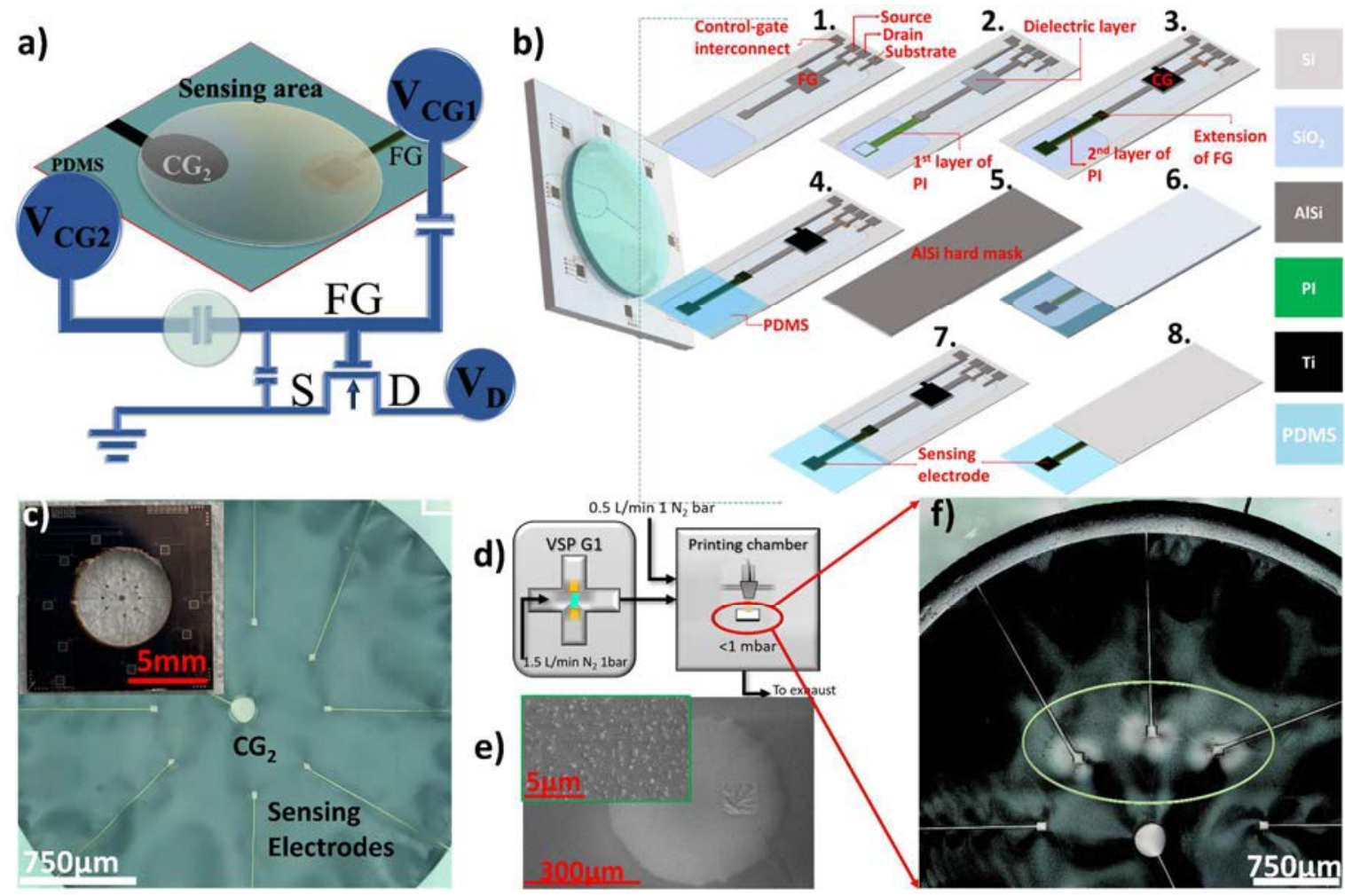

Figure 2: a) Schematic of the sensing mechanism, showing a section of the sensing area with $C_{2}$ and FG. The dualcontrol-gate configuration provides measurement of charges on the floating-gate extensions. b) Wafer-level fabrication of the dual-gate FG-FETs. c) Laser-optical microscope image of the frontside of a single chip. Second control-gate and sensing electrodes are visible. Inset shows the full chip after dicing. d) Sketch of the nanomaterial printer setup. e) SEM micrograph of local decoration of sensing electrodes with Au nanoparticle films (film morphology evidenced in the inset.) f) Backside micrograph of the chip by laser-optical microscopy, showing Au decoration on 3 of the 8 sensing electrodes.

selective material, including monitoring $\mathrm{pH}$ and neuronal activity [8], tracking the morphology of cancer cells and fibroblasts [12], and DNA hybridization [13]. However, Au's well-known incompatibility with CMOS process flows imposes its post-processing. Here, nanostructured, microscopically rough $\mathrm{Au}$ films were deposited by inertial impaction of a pure $\mathrm{Au}$ nanoparticle (NP) aerosol generated by spark ablation. The technique is mask-less (avoiding the issues of conducting lithography over substrates with high topography), spatially-selective, fast, and is conducted at room temperature. Moreover, while it does not presently allow batch wafer post-processing, it does not harm the flexible and suspended polymeric membranes supporting the electrodes, and yields surface roughness figures superior to other more cumbersome post-processing techniques (e.g. flow coating), let alone CMOS-compatible ones (e.g., e-beam evaporation, sputtering, atomic layer deposition).

\section{FABRICATION}

\section{Fabrication of the FET}

A 4-inch, $525 \mu \mathrm{m}$-thick, single-side polished p-type $\mathrm{Si}$ wafer was used for the fabrication of both nMOS- and pMOS-based sensors using a standard BiCMOS process (Fig. 2(b)). After defining source and drain terminals by ion implantation, a $200 \mathrm{~nm}$-thick gate oxide layer was thermally grown. A $6 \mu \mathrm{m}$-thick PECVD oxide layer was deposited and patterned on the wafer backside as etch hard mask. A 1000 nm-thick layer of AlSi was sputtered and patterned on the front side to implement electrical interconnects and floating gates (Fig. 2b(1)). A 170 nm-thick PECVD oxide layer was used as dielectric between $\mathrm{CG}_{1}$ and floating-gate electrodes. Polyimide (PI) was then used to sandwich most of the extension of the floating gate onto the $\mathrm{OoC}$ sensing region. This ensured electrical insulation and mechanical robustness, while leaving the sensing electrode in contact with the environment. Fig. $2 b(2)$ sketches the dielectric layer and first PI layer. A sputtered 300nmthick layer of Ti was patterned to serve as CGs and FG extensions. Then a second layer of PI was spin coated and patterned to complete the insulation of the FG extensions (Fig. 2b(3)). Polydimethylsiloxane (PDMS) was mixed with its curing agent in a 10:1 ratio, degassed, spin coated and cured to serve as $20 \mu \mathrm{m}$-thick membrane for the OoC cell culturing/charge sensing area (Fig. 2b (4)). After sputtering a $200 \mathrm{~nm}$-thick AlSi protection layer onto the PDMS (Fig. 2a(5)), the Si substrate was etched from the backside by deep reactive ion etching (DRIE) to land on the oxide layer (Fig. 2b (6)). The AlSi hard mask and residual silicon oxide were then removed by wet etching to complete the release of the PDMS membrane (front- and backside of a single sensor are sketched in Fig. 2b (7) and (8), respectively). The wafer was finally diced into 52 equal, square chips with footprint of $1 \mathrm{~cm}^{2}$, each chip containing 4 pMOS and 4 nMOS FETs (Fig. 2(c)).

\section{Post-processing for electrode surface structuring}

The Au NP aerosols were generated by a spark discharge generator (VSP G1) and deposited by means of a 

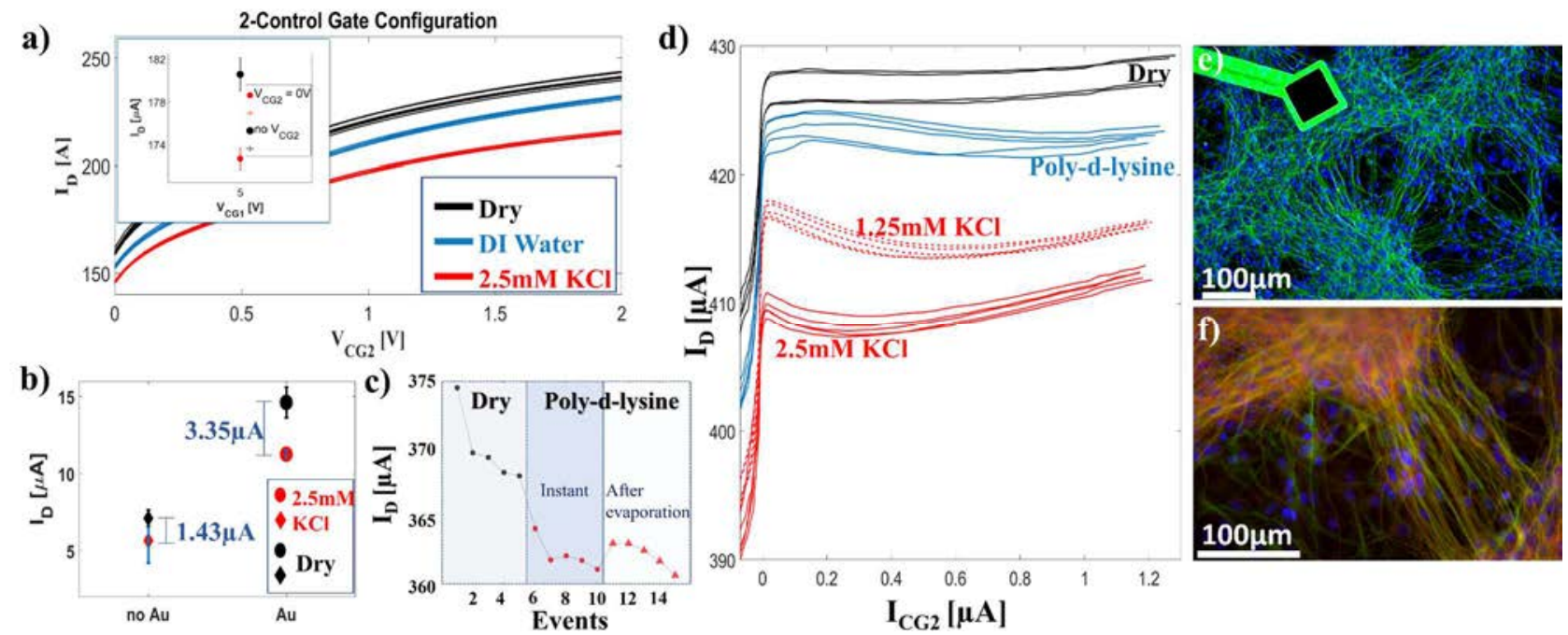

Figure 3: Characterization of nMOS-based sensors. a) Dual-gate configuration measurements without Au decoration of the sensing electrodes. Inset shows measurements in air while applying voltage through $\mathrm{CG}_{2}$ (red) and only using the single control-gate (black). b) Mean and standard deviation of multiple measurements on the same sensor, before and after Au decoration. The Au film increases the drain current shift for the same applied voltages (here, $V_{C G 1}=0 V$ and $V_{C G 2}$ $=2 \mathrm{~V}$ ). c) Consecutive measurements with the same sensor in air and with poly-d-lysine. d) $I_{D}$ shifts due to different liquids on Au-decorated sensor. Here, second control-gate voltages were swept (-2V to $2 \mathrm{~V})$ while applying constant voltage (5V) through the first control-gate. Comparison between dry (black), poly-d-lysine (blue) and KCl with different molarities (red). Culture test on the chips with hiPSC-derived cortical neurons. e-f) hiPSC-derived cortical neurons were stained for DAPI [e),blue], MAP2 [e), green] and synaptophysin [f], red] following 7 days of differentiation on chip.

nanoporous material printer (NMP) prototype developed by VSParticle B.V. (Fig. 2(d)). The NMP operates at $<1$ mbar pressure to create an ultrasonic gas jet with tunable diameter that transfers the NPs on the substrate using their inertia [14]. The deposition process used 99.999\% Au electrodes and $\mathrm{N}_{2}$ as carrier gas, resulting in pure and clean NP film surfaces and little waste production. A $5 \mathrm{~s}$ deposition resulted in $<500 \mu \mathrm{m}$ spot size.

Figs. 2(e) and 2(f) show successful damage-less completion of the Au post-processing step on the Ti sensing electrodes embedded in the $20 \mu \mathrm{m}$-thick PDMS membrane.

\section{MEASUREMENTS}

To characterize the sensors, a 4-probe measurement system connected to a semiconductor parameter analyzer was used to bias the FG-FETs and monitor changes in drain current $I_{D}$ ensuing from charges at the sensing electrodes.

\section{Measurements before electrode decoration}

As baseline test, $I_{D}$ values were recorded in air with and without applying voltage through $\mathrm{CG}_{2}$ with the same nMOS-based sensor (inset of Fig. 3(a)). Shift in the $I_{D}$ values demonstrate the electrical coupling between $\mathrm{CG}_{2}$ and FG electrodes. After characterization in air, a $2.5 \mathrm{mM} \mathrm{KCl}$ solution was introduced to the sensing area and consecutive measurements were recorded. The sensor was flushed and measurements with DI water were also recorded. The dualgate configuration shows unique $I_{D}$ characteristics for each measurement type, as evident from Fig. 3(a). We note that the absolute change in $\mathrm{I}_{\mathrm{D}}$ value is linearly proportional to the charge concentration of the solution, whereas the negative change is attributed to the difference in dielectric constants of the solutions and to the effect of coupling capacitance between $\mathrm{CG}_{2}$ and $\mathrm{FG}$.

\section{Measurements after electrode decoration}

Effect of Au decoration. For the same sensor used previously, measurements in air and with $2.5 \mathrm{mM} \mathrm{KCl} \mathrm{so-}$ lution before and after Au post-processing were recorded (Fig. 3(b)). The effect of electrode decoration is evident from changes in $I_{D}$ values. In addition, for the same applied voltage values, mean values of measurements after Au decoration show an up to 2.3 times increase in sensitivity.

Effect of membrane stress. After dry measurements, the $\mathrm{Au}$ decorated sensor was used to evaluate poly-d-lysine, a positively-charged polypeptide widely used in mammalian cell culturing [4] and stem cell research [15]. Fig. 3(c) shows shift in $I_{D}$ due to reversible binding of charges in $10 \mu \mathrm{L}$ poly-d-lysine $(0.1 \mathrm{mg} / \mathrm{ml})$. Interestingly, measurements after evaporation of the solution show similar $I_{D}$ values to those measured immediately after placing the solution at the sensing area. This suggests that possible membrane stress due to liquid handling did not notably affect the results, and confirms that the current shifts are primarily caused by charges bound on the electrodes.

SOT characterization: We conducted consecutive measurements of various solutions with Au-decorated FG extensions (Fig. 3(d)). First, a $\mathrm{KCl}$ solution $(2.5 \mathrm{mM}$ ) was introduced and measurements were recorded. The sensor was then flushed with DI water, and $\mathrm{KCl}$ with lower concentration $(1.25 \mathrm{mM})$ was introduced. Lastly, Poly-d-lysine was introduced on the sensing area. The sensor was flushed again and measurements were recorded. Changes in $I_{D}$ show differences accordingly with the different charge concentrations in each solution. The low leakage current from $\mathrm{CG}_{2}$ appears due to the reversable breakthrough of the native Ti oxide layer on top of the electrode, while applying voltage through $\mathrm{CG}_{2}$. 


\section{DISCUSSION}

We propose the following model to explain the increased sensitivity evidenced in the experimental results by virtue of $\mathrm{Au}$ electrode decoration. In the saturation regime of the transistor, the variation in the floating gate voltage $\Delta V_{F G}$ is expected to depend on the total capacitance of the device $C_{T O T}$ and on the variation of charges in close proximity to the sensing area $\Delta Q[5]$ :

$$
\Delta V_{F G}=\frac{C_{C G 1} V_{C G 1}}{C_{T O T}}+\frac{C_{C G 2} V_{C G 2}}{C_{T O T}}+\frac{Q_{0}}{C_{T O T}}+\frac{\Delta Q}{C_{T O T}}=\frac{\Delta Q}{C_{T O T}}
$$

Assuming a surface charge density $\sigma$ forming at the sensing electrode with surface $A_{S}$, then:

$$
\Delta Q=\int_{A_{S}} \Delta \sigma d s
$$

and $I_{D}$ can be related to the floating gate voltage as:

$$
\Delta I_{D} \cong a\left(\Delta V_{F G}-V_{T H}\right)^{2}
$$

where $V_{T H}$ is the threshold voltage and $a=\mu c_{o x} W / 2 L$ the FET transconductance, which depends on carrier mobility $(\mu)$, gate oxide capacitance per unit area $\left(c_{o x}\right)$, and on width (W) and length (L) of the gate. According to (3) a change in $I_{D}$ is thus in direct relation with the charge variation in close proximity to the sensing electrode. The deposition of the microscopically rough Au thin film increases the effective surface area $A_{s}$ and the available sites of the sensing electrode surface.

The proposed sensor avoids the cumbersome use of an external reference electrode. While the control over the charging of the surface is not direct [11], the electrode surface enhancement method, coupled with the novel dualgate control configuration here demonstrated, enables higher charge accumulation on the sensing electrode, thanks to the combination of electrical field between $\mathrm{CG}_{2}$ and FG and larger effective surface area.

\section{Cell Culture \& Differentiation}

On chip biocompatibility and neuronal differentiation tests were conducted by culturing and differentiating human-induced pluripotent stem cell (hiPSC)-derived cortical neurons. Levels of potassium in the brain higher or lower than its threshold value might cause different disease phenotypes such as epilepsy [16] or chances of developing a headache [17]. Therefore, the ability to sense potassium from neuronal cultures in vitro is critically needed for brain disease modelling.

Neural Progenitor Cells (NPCs) were successfully differentiated on the chips into cortical neurons for 7 days, with staining showing mature neuron marker (MAP2/green) as well as the production of synaptic vesicle (synaptophysin/red) (Fig. 3(e-f)). Long neurite extensions are visible as well as distinct network formation, indicating that the chip is suitable for live measurements using hiPSCderived cortical neurons

\section{CONCLUSION}

A FET-based electrochemical sensor with enhanced sensitivity and selectivity to chemical species was integrated by a mostly CMOS-compatible fabrication process within a microelectromechanical $\mathrm{OoC}$ device. The dualgate control architecture of the FETs allows higher sensitivity compared to single capacitively-coupled control-gate FETs. In addition, spark ablation was used to alter the sur- face morphology of the sensing electrodes with thin nanoporous Au films. This post-processing method allows fast and spatially-selective patterning over topographical substrates without the need for cumbersome and time-consuming lithography steps, and without damaging the fragile and biocompatible polymer membranes. As a result, more than doubled sensitivity was achieved, and the sensor could be used to successfully identify different SOTs, including low-concentration $\mathrm{KCl}$ solutions and poly-D-lysine.

Furthermore, culture tests showed that NPCs were successfully differentiated to cortical neurons on our chip, and visual inspection was possible due to the optical transparency of the culture area. These results suggest that our MEM OoC device can be employed in the near future for testing the potassium level of human brain cells (whereby normal levels of $\mathrm{K}^{+}$are $150 \mathrm{mM}$ intracellular and $5 \mathrm{mM}$ extracellular), a crucial indicator for the wellbeing and functioning of the brain [18].

\section{ACKNOWLEDGEMENTS}

The authors thank the staff at the Else Kooi Laboratory of TU Delft and VSParticle B.V. for their support and for enabling us to use the Proto-0 NMP in our lab.

This work was supported by the Netherlands Organon-Chip Initiative, an NWO gravitation project funded by the Ministry of Education, Culture and Science of the government of the Netherlands (024.003.001), and by the NWO Nano Copper for Power Electronics Interconnection Project 729.001.023

\section{REFERENCES}

[1] U. Marx et al., ALTEX 33(3), pp. 272-321, 2016.

[2] M. Mastrangeli et al., ALTEX36(4), p. 650-668, 2019.

[3] M. S. Thomas et al., J. Phys. Chem. Lett. 9(6), pp. 1335-1339, 2018.

[4] B. Chen et al., IEEE Sensors Journal, 11(11), pp. 2906- 2910, 2011.

[5] A Spanu et al., Scientific reports, 5, 8807, 2015.

[6] H. Aydogmus et. al., IEEE Sensors 2020.

[7] P. Fromherz et al., Science, 252, pp. 1290-1293, 1991.

[8] S. Martinoia et al., Biosensors and Bioelectronics, 16(9-12), pp. 1043-1050, 2001.

[9] A. Loi et al., Appl. Phys. Lett. 86(10), 103512, 2005.

[10] M. Kaisti. Biosensors and Bioelectronics, 98, pp. 437448, 2017.

[11]M. Kaisti et al., IEEE Trans. Electron Dev., 62.8, pp. 2628-2635, 2015.

[12] P. Lin et al., Adv. Mater. 22.33, pp. 3655-3660, 2010.

[13] M. H. Park et al., J. Phys. Chem. C 120.9, pp. 4854 4859, 2016.

[14] Schmidt-Ott, A. (Ed.). Spark Ablation: Building Blocks for Nanotechnology. CRC Press, 2019.P.

[15] P. Rocheteau et. al., Nature communications, 6(1): 1$12,2015$.

[16] C. Simons et. al., Nature Genetics, 47, pp.73-77, 2015.

[17] Z. Guo, et al., Eneuro, 6(4), 2019.

[18] G. Florence et. al., Communications in Nonlinear Science and Numerical Simulation, 17(12), pp. 47004706, 2012.

CONTACT

*H. Aydogmus, h.aydogmus@tudelft.nl 